\title{
Atomized spray droplet size prediction from multi-hole nozzle for direct injection gasoline engines
}

\author{
Motoyuki ABE*, Eiji ISHII* and Hideharu EHARA** \\ ${ }^{*}$ Research \& Development Group, Hitachi Ltd. \\ 832-2 Horiguchi, Hitachinaka-shi, Ibaraki 312-0034, Japan \\ E-mail:motoyuki.abe.kc@hitachi.com \\ **Powertrain \& Electronic Control Systems Div., Hitachi Automotive Systems, Ltd. \\ 2520 Takaba, Hitachinaka-shi, Ibaraki 312-8503, Japan
}

Received: 17 March 2017; Revised: 12 May 2017; Accepted: 7 June 2017

\begin{abstract}
We investigated atomized spray droplet size from multi-hole nozzles for direct injection gasoline (DIG) engines. Our findings showed that the droplet size can be described by the nozzle-hole geometry. For the DIG engine, adequate spray pattern and finely atomized spray are important to achieve low emission or/and low fuel consumption. As an injector for DIG engines, multiple holes (multi-hole) type nozzle is typically used because of adaptability of spray pattern. The multi-hole type spray has been generally used for Diesel engines, and the characteristics have been investigated in earlier studies. However, multi-hole nozzles for DIG engines require narrow spacing as fuel passage just upstream of the orifice hole. The narrow fuel passage affects spray characteristics including droplet size. This makes droplet size prediction and designing orifice geometry difficult, thus the narrow passage geometry needs to be incorporated as a design parameter. We therefore investigated relation between droplet size and nozzle geometry both experimentally and theoretically. Experimentally, we evaluated an experimental dataset which was done in previous work in which the spray droplet size from the fabricated test sample nozzles, and determined the relationship parameters between nozzle geometries, flow rate, and droplet size. The experimental result in which its pressure range was 1 to $15 \mathrm{MPa}$ showed that velocity at outlet of orifice-hole is a dominant factor to determine droplet size, and that the velocity has a correlation with the nozzle geometry. Theoretically, we focused on a pressure-drop at the narrow passage, which can describe velocity at the outlet of the orifice-hole. Finally, theoretical approach described the droplet size by using the orifice geometries incorporating the parameter of narrow passage.
\end{abstract}

Key words : Direct injection gasoline engine, Fuel injector, Multi-hole nozzle, Atomization, Droplet size

\section{Introduction}

The direct injection gasoline (DIG) system is one approach to reduce fuel consumption (Iwamoto, et al, 1997) for spark ignition engines, which allows large compression ratio of engine cycle by cooling effect of fuel vaporization in combustion chamber. Important issues on the DIG engine are hydrocarbon (HC) emission and particulate matter (PM) emission. In order to achieve low fuel consumption and low HC emission, a fuel injector is a key component which determines the performance of a direct injection gasoline (DIG) engine(Tanimura, et al, 1997 and Ueda, et al, 2000).

Dominant causes of HC/PM emission are coarse droplets and fuel adhesion onto the surface of combustion chamber, i.e., a cylinder wall, piston surface, or intake/exhaust valves. In a DIG system, the injector nozzle sprays fuel directly into the combustion chamber, in order to prevent the fuel adhesion, the fuel spray pattern should be matched to the combustion chamber geometry. This is the reason of that multi-hole injectors are used for DIG engines because multiple holes nozzle enables adaptable spray pattern. Further the spray droplets need to be finely atomized to promote the evaporation of fuel before ignition. The HC/PM are generated from high concentration region of fuel mixture which appears around coarse droplet remaining as liquid phase. Finely atomized droplet enables to be vaporized fast and to be mixed with air, which may eliminate highly concentrated region. There is therefore a need for a fine atomization 
nozzle.

Therefore, in order to design fine atomization nozzles, prediction method of droplet size (or atomization level) should be clarified. Prediction methods has already been reported for simple plain hole orifices (Tanasawa, et al., 1951 and 1957, Kurabayashi, et al., 1995). These reports say that droplet size can be described by velocity in the orifice hole. Atomization form and relation between the droplet size and the velocity were classified by using the velocity range. For low velocity range, which is for port fuel injectors, is classified as a "wavy-jet" (Tanasawa, et al., 1951) atomization form, and its relations between atomization level and nozzle dimensions have already been investigated (Varde, et al., 1999, Ren, et al., 2001 and Tani, et al., 2000). Also diesel nozzles have been investigated in atomization (Koo, et al., 1990 and Lai et al., 1998), it is classified as a "completely sprayed" (Tanasawa, et al., 1951) atomization form.

However, the velocity range for DIG nozzles is classified as "partially sprayed" (Tanasawa, et al., 1951) atomization form, because of its lower outlet velocity compared with diesel nozzle. In addition, typical injector for DIG engines is designed as direct-driven solenoid actuator. This type of actuator should have shorter valve stroke compared with hydraulic-driven actuator (i.e. diesel nozzle), which brings narrow fuel passage (thin gap) around orifice hole. Generally, the gap affects flow motion and phenomena around the orifice hole, it might affect atomization process. Thus authors have investigated the DIG nozzles experimentally, and found that a coefficient of relation between velocity and droplet size is different from diesel nozzle (Abe, et al, 2013). Further, the previous study showed that droplet size from DIG nozzles can be described by using geometry factors around orifice.

Objective of this study is to describe prediction methodology of atomization on DIG nozzles. This study reveals theoretical discussion regarding geometry effect including the narrow fuel passage around orifice hole. Further, the theoretical approach will be reviewed with experimental result.

\section{Structure of multi-hole injector for DIG}

A typical solenoid injector for DIG (Abe, et al, 2013) is described in Fig.1 as sectional image, and its structure around orifice is shown in Fig.2. In an operation state, pressurized fuel is supplied from a fuel-rail, and is filled in the injector (Fig.1). An armature and valve-body (needle valve) can be operated by magnetic force between armature and magnetic-core. This magnetic force exerted on the armature opens directly the needle valve against pressurizing force. As the magnetic force decreasing by increasing a gap between the armature and the core, the valve stroke (lift) should be limited (typically, 40-100 micro meters). This is a typical injector for gasoline direct injection, and is different from high pressure diesel injection system which is operated by actuator driven hydraulic force. This difference makes the limitation of the valve lift.

The tip of the nozzle (Fig.2) equips multiple (typically 5-7 holes) orifices. Spray beams from this type nozzle can generate pre-designed spray pattern steadily compared with swirl-type nozzle which generates hollow-cone pattern. Direction for each orifice determines the spray beam direction. The orifices are drilled from inner tapered valve-seat surface to outer nozzle tip, and the tapered surface has a sealing valve seat at just upstream of the orifice. The needle valve contacts between the valve seat in closing state. The needle valve has a protrusion at end of the needle, and that protrusion fills volume of the tapered pit at downstream of the valve seat. In opening state after lifting the needle valve, a fuel passage gap (seat-gap) appears between the valve seat and the needle surface. The pressurized fuel in delivered into the orifices through the seat-gap, the fuel comes out from the orifice as spray.

According to previous experimental investigations (Abe, et al, 2013), spray characteristics (droplet diameter and flow rate) are mainly determined by area of the gap and total section area of the orifices. The area of the seat-gap is determined by diameter of valve-seat and angle of the tapered valve seat, which is geometrically described as equation (1). The total orifices area described in equation (2) is determined by orifice diameter and number of orifices. Where, $A_{\text {gap }}$ is area of the seat-gap, $A_{\text {orifice }}$ is the total section area of orifices, $D_{\text {orifice }}$ is the orifice diameter, $D_{\text {seat }}$ is the valve-seat diameter, $\phi_{\mathrm{s}}$ is angle of the tapered valve seat, $Y_{\text {lift }}$ is maximum lift (stroke) of the injector and $N$ is number of orifices.

$$
\begin{gathered}
A_{\text {gap }}=\pi D_{\text {seat }} \cdot Y_{\text {lift }} \cdot \cos \left(\frac{\phi_{s}}{2}\right) \cdot \sin \left(\frac{\phi_{s}}{2}\right) \\
A_{\text {orifice }}=\frac{\pi \cdot N \cdot D_{\text {orifice }}^{2}}{4}
\end{gathered}
$$




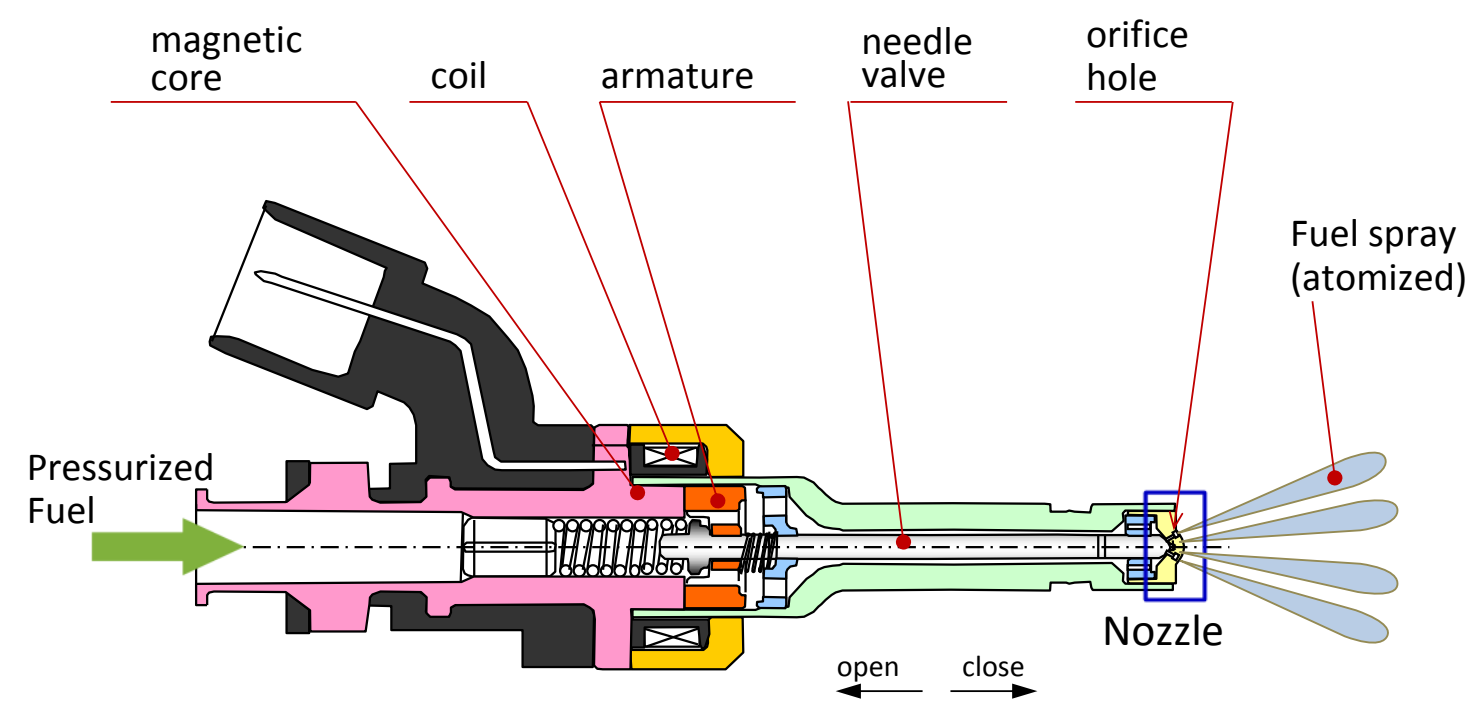

Fig. 1 Solenoid driven injector for direct injection gasoline engines. A typical sectional image is shown. Arrows indicates directions of needle-valve movement. A Box shows a location of nozzle structure in which detail is illustrated in Fig.2

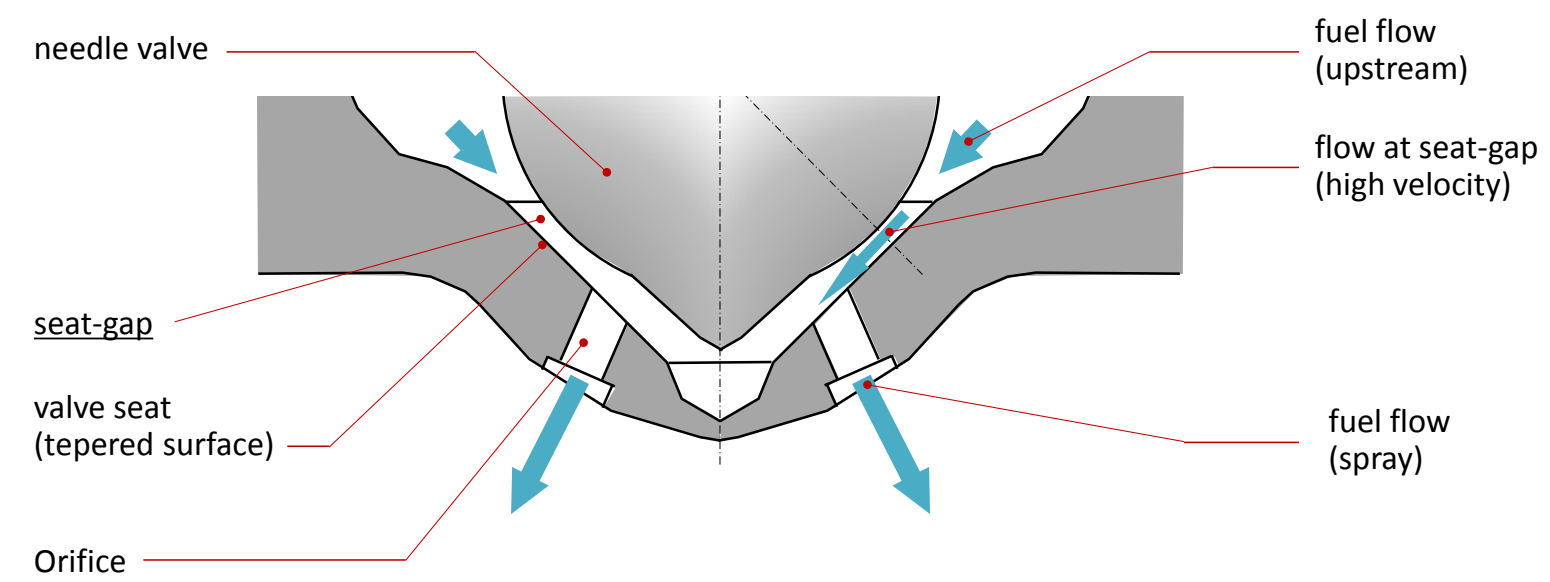

Fig. 2 Detailed structure around orifice holes. This figure shows a magnified and 90 degrees rotated image of boxed area in Fig.1.

\section{Theoretical discussion of flow around orifices}

Fuel flow around the orifices affect is discussed in this chapter by theoretical approach. According to previous experimental investigation (Abe, et al, 2013) has showed that fuel velocity in orifice can be described by using ratio of the seat-gap and the orifices area, where the ratio $K$ (passage area ratio) is defined as equation (3).

$$
K=A_{\text {seat }} / A_{\text {orifice }}
$$


The Fig.3 describes definitions of physical values around the seat-gap and orifices, where $P_{\mathrm{f}}$ is fuel pressure at upstream of the valve-seat, $P_{0}$ is ambient pressure at downstream of the orifice, $v_{\text {seat }}$ is averaged velocity at the seat-gap and $v_{\text {orifice }}$ is averaged velocity at inside of the orifice. Relation between these velocities and geometry around orifice is described as following equation by continuity relation, so that the seat-gap velocity $v_{\text {seat }}$ can be described by $v_{\text {orifice}}$.

$$
\begin{aligned}
& A_{\text {seat }} v_{\text {seat }}=A_{\text {orifice }} v_{\text {orifice }} \\
& v_{\text {seat }}=\frac{A_{\text {orifice }}}{A_{\text {seat }}} v_{\text {orifice }}=\frac{1}{K} v_{\text {orifice }}
\end{aligned}
$$

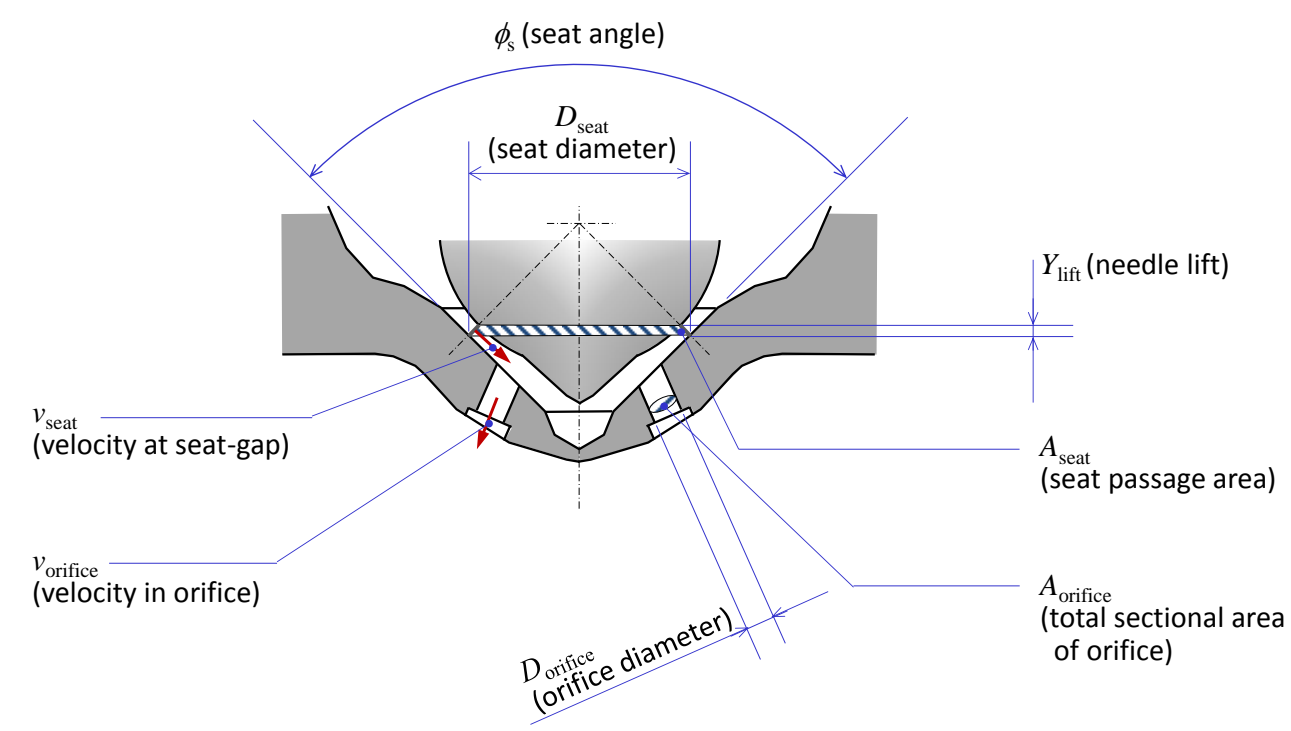

Fig. 3 Definitions of physical values around the nozzle structure, which includes both geometric parameters and velocities at important locations.

As a point of view regarding pressure drop at both of the valve-seat and the orifices, pressure difference $P=P_{\mathrm{f}}-P_{\mathrm{o}}$ becomes kinetic energy at the orifices and dissipated energy as pressure loss at both the valve-seat and the orifice. As the pressure losses can be described that they are proportional to powered value of velocities. High velocity causes large pressure loss, thus narrow fuel passage becomes dominant factor for the pressure loss. In the DIG solenoid injector, there are two main narrow fuel passages which are orifices and seat-gap. Therefore, the relation of the pressure difference and velocities can be expressed as following equation (5) which describes summation of kinetic energy (corresponding to $v_{\text {orifice}}$ ) at orifice outlet and energy losses (corresponding to both $v_{\text {orifice }}$ and $v_{\text {seat }}$ ) at upstream of the outlet, where $\rho$ is density of fuel, and $\lambda_{1}, \lambda_{2}$ are pressure loss coefficients.

$$
P=P_{f}-P_{0}=\frac{1}{2} \rho v_{\text {orifice }}^{2}+\frac{\lambda_{1}}{2} \rho v_{\text {orifice }}^{2}+\frac{\lambda_{2}}{2} \rho v_{\text {seat }}^{2}
$$

Here, equation (4) can be substituted for equation (5), the averaged velocity in the orifice describes pressure difference as following equation.

$$
\frac{2 P}{\rho}=\left(1+\lambda_{1}+\frac{\lambda_{2}}{K^{2}}\right) v_{\text {orifice }}^{2}
$$


In the equation (6), its coefficients $\lambda_{1}, \lambda_{2}$ could be determined by experiment or simulation as constant values. Thus the equation (6) indicates that the passage area ratio $K$ which is geometrically determined can describe averaged effective velocity in the orifice when the pressure drop $P$ is determined. The velocity in the orifice $v_{\text {orifice }}$ can be expressed as following equation (7), where $f(K)$ is a function which determines the pressure loss coefficient. The velocity at inside of orifices is proportional to square root of pressure difference, and its coefficient depends on the ratio between sectional area of orifices and area of seat-gap. Therefore, when the $v_{\text {orifice }}$ is normalized by square-root of pressure drop $P$, the normalized velocity can be described as the function $f(K)$.

$$
v_{\text {orifice }}=\sqrt{P} \cdot f(K)=\sqrt{P} \cdot\left(\frac{2}{\rho}\right)^{0.5}\left(1+\lambda_{1}+\frac{\lambda_{2}}{K^{2}}\right)^{-0.5}
$$

\section{Discussion on experimental result}

Experimental results regarding relation between nozzle geometry and velocity are reviewed in this chapter. The experiments have been disclosed in previous investigation, this chapter re-plots the experimental data by using the relation between velocity and geometric factor, which has been discussed in previous chapter. This relation will be confirmed and its coefficients will be determined experimentally in this chapter.

Further, this chapter discusses regarding atomization characteristics. It is well known that atomization characteristics and its process are strongly related to the velocity in orifices. Thus we investigated experimental relation between atomization level and estimated velocity. Finally this chapter will introduce an enhancement of the experimental relation into prediction method which allows estimating atomization level by only using geometric information of injector nozzle.

According to the equation (7), the velocity can be normalized by square root of the pressure difference between up/downstream of the orifice. The normalized velocity is described as equation (8).

$$
\frac{v_{\text {orifice }}}{\sqrt{P}}=\frac{Q_{\text {st }}}{A_{\text {orifice }}}
$$

The experimental results which were taken in previous work (Abe, et al, 2013) and 40 plots have been added to ensure their relation in Fig.4 with predicted line by equation (6), where velocity in vertical axis is normalized by pressure under which static flow rate defined. The experimental plots include result from samples with different orifice diameter and different valve lift. The diameters were from 0.15 to $0.29 \mathrm{~mm}$, and the number of orifice was six. The valve lifts were from 25 to $83 \mu \mathrm{m}$, the seat diameter was $1.4 \mathrm{~mm}$, and the seat angle was 90 degree (this configuration corresponds to a combination of $2 \mathrm{~mm}$ spherical ball-valve and right cone angle tapered valve-seat).

The values of normalized velocity plotted in Fig.4 were estimated by using static flow rate $Q_{\text {st }}$ of each injector prototype experimentally under rail-pressure $P$ of $10 \mathrm{MPa}$ (A flow which is displacement-counter type was used to measure the flow rate). In the experiment, Law Aromatic White Spirit (LAWS) was used as fluid, which the density $\rho$ was $694 \mathrm{~kg} / \mathrm{m}^{3}$. The drawn line is calculated by using equation (7) and (8) with geometric factor $K$. For coefficients $\lambda_{1}$ and $\lambda_{1}$ regarding pressure losses, they might be affected by geometric factors around orifices, but Fig. 4 shows that they can be considered as constant values for various valve-lift and orifice diameter (these geometric factors were varied in the applied data set, angle of orifice hole was 45 degree from valve axis, and seat angle was 90 degree). The experimental plots brought pressure-drop coefficients $\lambda_{1}$ and $\lambda_{2}$, which were determined by least-square method. The experimentally determined values were $\lambda_{1}=2.5$ and $\lambda_{2}=1.5$.

The plot shows acceptable prediction of velocity by equation (6), thus the experimental relation regarding velocity has been described by theoretical formula with coefficients determined by the experimental result. According to Fig.4, good agreement can be observed from $K=0.3$ to 1.8 . This is considered that the theoretically introduced relation between geometric factor $K$ and velocity in orifice $v_{\text {orifice }}$ can be applied to wide range of the geometric factor of 
injector.

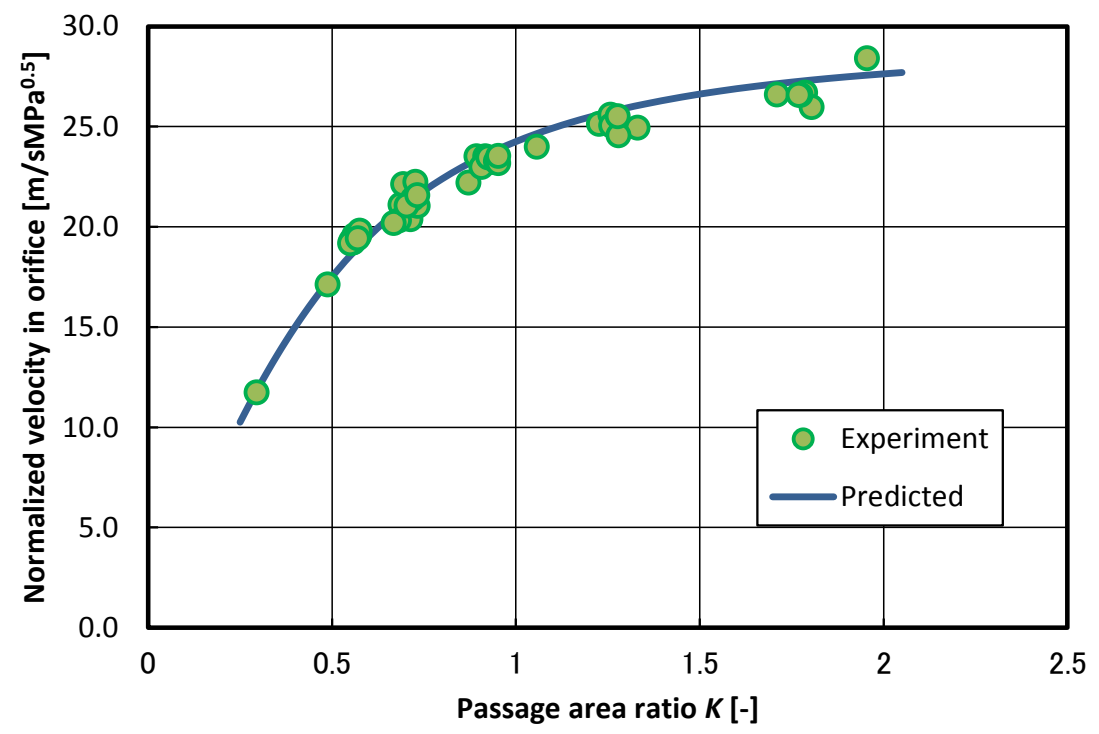

Fig. 4 Experimental result of averaged velocity at orifice. The velocity on vertical axis was normalized by square root of pressure. The horizontal axis indicates passage area ratio $K$ which is ratio between seat-gap area and orifices. The experimental data is previously investigated result (Abe,et al), the predicted line is added by using relation of equation (6) with coefficients determined by least mean square method experimentally.

The velocity prediction can be extended into atomization prediction because Sauter-mean droplet diameter (SMD) from multi-hole DIG injector depends on velocity in the orifice. The relation between the velocity and SMD can be described by an equation (9) (Abe, et al, 2013). According to previous discussion, the velocity can be described as a function of geometric factor $K$. This means that geometric information can describe atomization level. Thus, an equation (10) has been introduced as a combination of equation (7) and (9). The SMD should be predicted by the factor $K$ and rail-pressure $P$.

The Fig.5 shows experimental result which has already been investigated in previous work and the experimental coefficients were determined that constant $C$ was $5.5 \times 10^{3}$, index $p$ was 1.5 (Abe, et al, 2013). The experimental SMD were measured by LDSA, in which its injection operation was done in intermittent condition and overall averaged droplet size was adopted. The measured data may contain transient condition of velocity at nozzle, but the transient (lower velocity than steady condition) duration was less than $2 \%$ of total injection duration, so that effect of transient state for injection mass is considered further less to omit the transient effect.

Further the Fig.5 shows a predicted line by using equation (10), and good agreement can be observed in its tendency. The experimental plots have variance from the predicted line, which is considered that caused by effect of internal flow condition in the orifice, i.e. large separated flow and its vortex may cause effect to the first break-up of liquid stem to droplet at the orifice outlet for example. The equation (10) expresses SMD by using geometric factor $K$ (passage area ratio of seat and orifices), which can be used for droplet size estimation for injectors with narrow gapped passage area around inlet of orifice, i.e. multi-hole type DIG injectors, although orifice diameter is one of factors to determine droplet size in general. It is considered that the diameter is an indirect parameter in "partially sprayed" or "complete sprayed" range of velocity (classified by Tanasawa, et al, 1951) because ratio of kinetic energy and surface tension energy (i.e. Weber number) is a dominant factor, so that effect of hole diameter difference could be expressed as variation of velocity (The diameter is one of a parameter to determine the velocity) in the equation (9).

$$
\begin{aligned}
& D_{32}=C v_{\text {orifice }}^{-p} \\
& D_{32}=C\left\{\left(\frac{2 P}{\rho}\right)^{0.5}\left(1+\lambda_{1}+\frac{\lambda_{2}}{K^{2}}\right)^{-0.5}\right\}^{-p}
\end{aligned}
$$


This relation indicates that the orifice work as diffuser for fuel fluids just after the narrow gap so that its velocity should be reduced in the orifice. In order to obtain finer atomization with same static flow of an injector, larger $K$ (i.e. faster $v_{\text {orifice}}$ ) could be better, which is larger area of valve-seat passage.

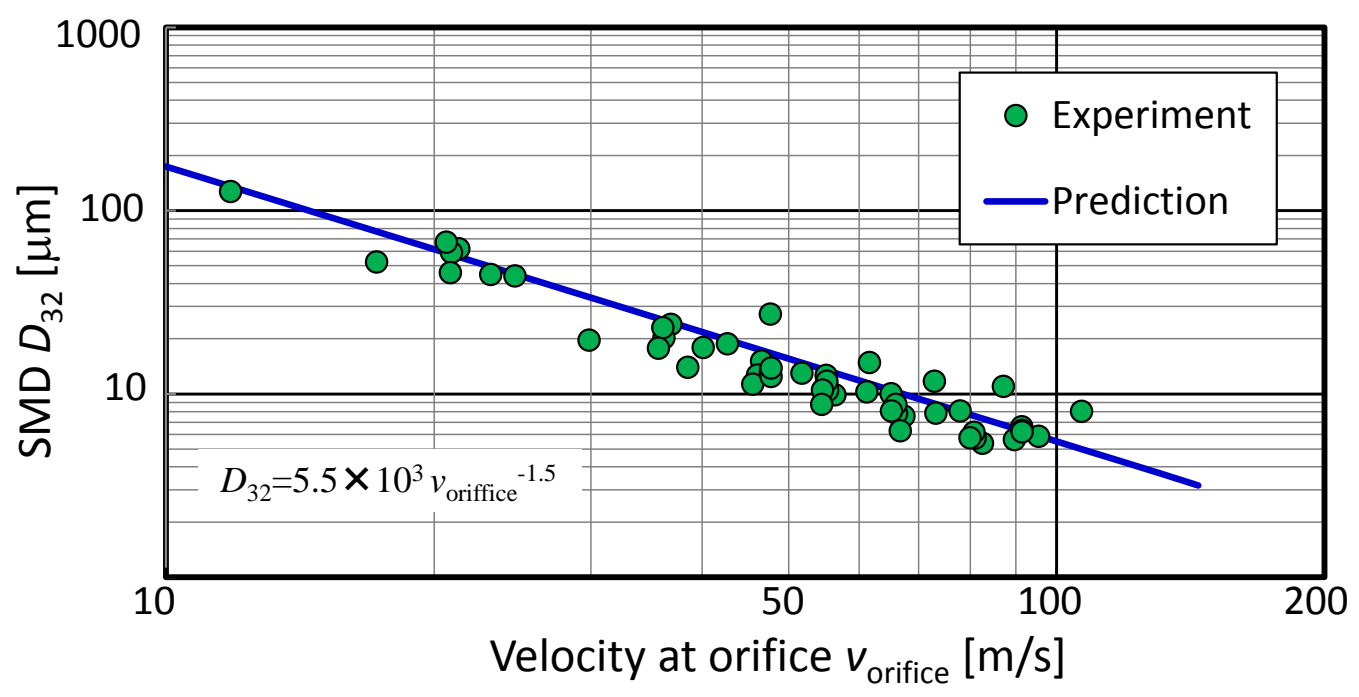

Fig. 5 Comparison between experimental and prediction result of SMD. Equation (9) was used to predict droplet size by using $K$ which is the geometry factor of DIG nozzle. Where droplet size measurement was done by laser diffraction droplet-size analyzer (LDSA), and LAWS was used as fuel. The fuel pressure for the experiment was set from $1 \mathrm{MPa}$ to $15 \mathrm{MPa}$, injectors as specimen were same as velocity prediction.

\section{Conclusions}

Atomization by multi-hole nozzles of fuel injector for DIG engines has been investigated. The DIG injectors require narrow spacing as fuel passage just upstream of the orifice hole, where the passage affects spray characteristics including droplet size. In this study, fluid velocity in nozzle orifice was discussed theoretically. Further, we applied the velocity theory into experimentally measured droplet size results in which the measurement was done in range of 1 to $15 \mathrm{MPa}$ (corresponding velocity range of 12 to $106 \mathrm{~m} / \mathrm{s}$ ), and concluded as followings.

1. The velocity in nozzle orifice of DIG injector can be described as an equation by using geometric factor $K$ which is ratio of the orifice area and the narrow passage area. Its coefficients of the equation were determined through experimentally obtained velocity information. Finally, equation for velocity prediction has been introduced.

2. Droplet size prediction method for DIG has been disclosed by combining the velocity prediction and experimental result of atomization. Experimentally obtained droplet-size as SMD has been reviewed and the predicted result was shown. Finally we concluded that the geometric factor of fuel passage area ratio $K$ is one of important factors to predict droplet size of DIG injectors. 


\section{References}

Abe, M., Ehara, H., Ishikawa, T., Soma, M., Spray atomization study on multi-hole nozzle for direct injection gasoline engines, SAE, 2013-01-1596 (2013)

Iwamoto, Y., Noma, K., Nakayama, O., Yamauchi, T. and Ando, H., Development of Direct Injection Gasoline Engine, SAE970541 (1997).

Koo, Ja-Ye, Martin, J. K., Droplet Sizes and Velocities in a Transient Diesel Fuel Spray, SAE900397 (1990)

Kurabayashi, T., Atomization Technology, IPC, p 29 (1995).

Lai, M.C., Thomas, T.C., Wang, et al., Microscopic Characterization of Diesel Sprays at VCO Nozzle Exit, SAE982542 (1998)

Ren, Wei-Min, Sayar, H., Influence of Nozzle Geometry on Spray Atomization and Shape for Port Fuel Injector, SAE2001-01-0608(2001)

Tanimura, Y., Takeuchi, K., Terada, T., Sugiura, S. and Katayama, Y., Compact Magnetic Solenoid Valves Using a Composite Magnetic Material, SAE970852, (1997).

Tanasawa, Y., Toyota-Technology, 5-5 (1951).

Tanasawa, Y., Theory of Fuel Injection, Sankaido (1957).

Tani, Y., Mori, Y., Mochizuki, K., Suzuki, A., Fuel Atomization of a Multiple-hole Nozzle Injector, SAE2000-01-1428(2000)

Ueda, S., Mori, Y., Iwanari, E., Oguma, Y. and Minoura, Y., Development of a New Injector in Gasoline Direct Injection System, SAE2000-01-1046 (2000).

Varde, K. S., Popa, D. M., Varde, L. K., Multiple-hole Nozzle Atomization for SI Engines, SAE1999-01-0564(1999) 